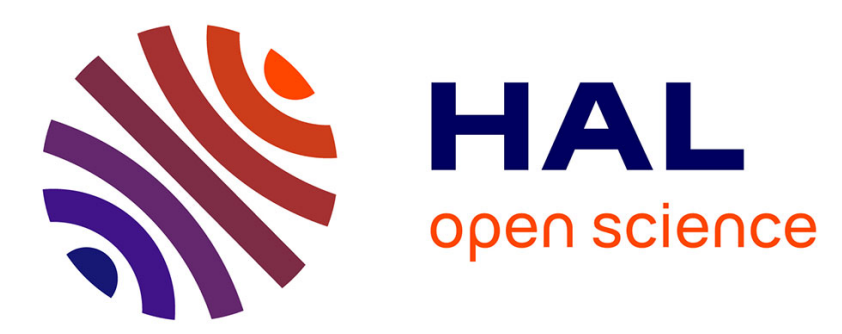

\title{
Tenue mécanique de fenêtres de saphir sous hautes pressions hydrostatiques
}

\author{
J. Fournier, J.M. Besson
}

\section{To cite this version:}

J. Fournier, J.M. Besson. Tenue mécanique de fenêtres de saphir sous hautes pressions hydrostatiques. Revue de Physique Appliquée, 1970, 5 (2), pp.333-335. 10.1051/rphysap:0197000502033300 . jpa00243397

\section{HAL Id: jpa-00243397 https://hal.science/jpa-00243397}

Submitted on 1 Jan 1970

HAL is a multi-disciplinary open access archive for the deposit and dissemination of scientific research documents, whether they are published or not. The documents may come from teaching and research institutions in France or abroad, or from public or private research centers.
L'archive ouverte pluridisciplinaire HAL, est destinée au dépôt et à la diffusion de documents scientifiques de niveau recherche, publiés ou non, émanant des établissements d'enseignement et de recherche français ou étrangers, des laboratoires publics ou privés. 


\title{
TENUE MÉCANIQUE DE FENÊTRES DE SAPHIR SOUS HAUTES PRESSIONS HYDROSTATIQUES
}

\author{
J. FOURNIER et J. M. BESSON \\ Laboratoire de Physique des Solides de la Faculté des Sciences de Paris, France, \\ Equipe de Recherche associée au C. N. R. S.
}

(Reçu le 17 décembre 1969)

\begin{abstract}
Résumé. - Nous présentons des résultats sur la tenue jusqu'à 17000 bars de fenêtres de saphir. Ce matériau résiste aux compressions et décompressions brutales jusqu'à cette pression pour des épaisseurs moindres que celles qui sont généralement utilisées. Les dimensions des montages optiques haute pression peuvent ainsi être allégées et l'ouverture optique considérablement augmentée.

Abstract. - Data are presented on the behavior of sapphire windows under 17000 bars pressure. Those windows accept fast pressure changes up to this pressure for less thickness than is generally thought necessary. It is thus possible to build lighter optical vessels with larger optical aperture.
\end{abstract}

I. Introduction. - Il existe une très grande variété de matériaux utilisables comme fenêtres optiques sous hautes pressions. La majorité de ces matériaux [1] ne sont utilisables qu'avec des précautions qui en rendent l'emploi difficile. Le diamant et le saphir se distinguent, au contraire par une tenue excellente ce qui permet de réaliser des fenêtres d'épaisseur faible et de grande ouverture. Nous rapportons ici des résultats sur des essais de fenêtres de saphir. Ces essais montrent que la limite supérieure d'emploi de ce matériau est beaucoup plus élevée que celle qu'on peut déduire des résultats publiés jusqu'ici.

Le saphir blanc synthétique est transparent de $1800 \AA$ à 5,5 microns environ. C'est donc un matériau optique utilisable dans une grande gamme de longueurs d'onde. Pour l'utilisation sous fortes pressions, la méthode la plus communément employée est celle du joint de Poulter où une fenêtre cylindrique de diamètre $D$ et d'épaisseur $e$ est appuyée par la pression sur un siège métallique plan percé d'un orifice de diamètre $d$. Pour une pression donnée on a intérêt à majorer les rapports $d / e$ et $d / D$ afin d'augmenter l'ouverture optique équivalente et de diminuer l'encombrement de l'obturateur et donc le poids de la bombe optique.

Nous présentons ici quelques résultats sur :

- l'étanchéité du joint de Poulter,

- les performances de fenêtres jusqu'à 17000 bars,

- la tenue de joints à encastrement baguant la fenêtre, jusqu'à la même pression.

Ces résultats n'ont aucun caractère systématique mais peuvent permettre néanmoins la réalisation d'ensembles optiques beaucoup plus compacts que ceux décrits jusqu'ici.

II. Description des ensembles. - Deux types d'obturateurs optiques ont été utilisés. La figure 1 donne pour mémoire le schéma d'un joint classique de

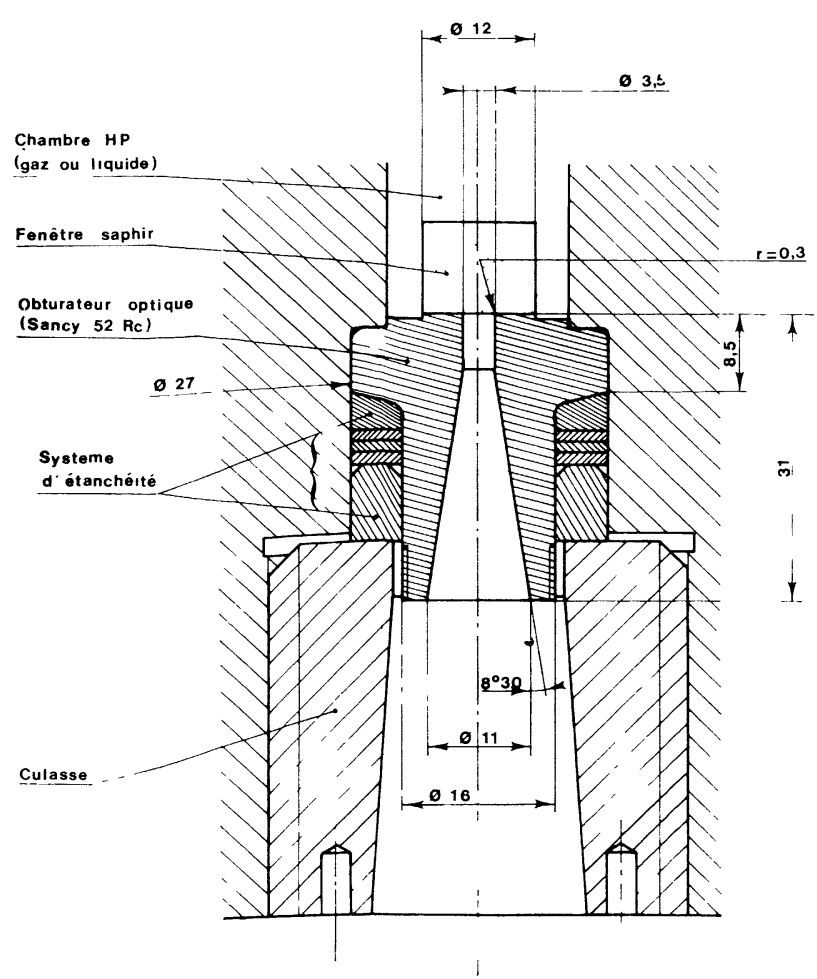

FIG. 1. - Joint classique de Poulter sur un obturateur de Bridgman. 
Poulter sur un obturateur de Bridgman, les dimensions de la fenêtre sont :

$$
\begin{aligned}
D & =12 \mathrm{~mm} \\
e & =9,5 \mathrm{~mm} \\
d & =3,5 \mathrm{~mm} .
\end{aligned}
$$

L'axe cristallin est parallèle à l'axe du cylindre. Ces fenêtres sont très largement surdimensionnées, comme nous le montrons au paragraphe III.

Le montage de la figure 2 comprend un empilement de bagues à encastrement sur la génératrice de la fenêtre elle-même. L'empilement utilisé est, dans l'ordre : Polyuréthane-Téflon-Bronze ELCO, la dernière bague enserrant à la fois la fenêtre et le siège métallique. Les bagues de bronze et de téflon supérieures sont des cales qui assurent un préserrage doux avant la montée en pression.

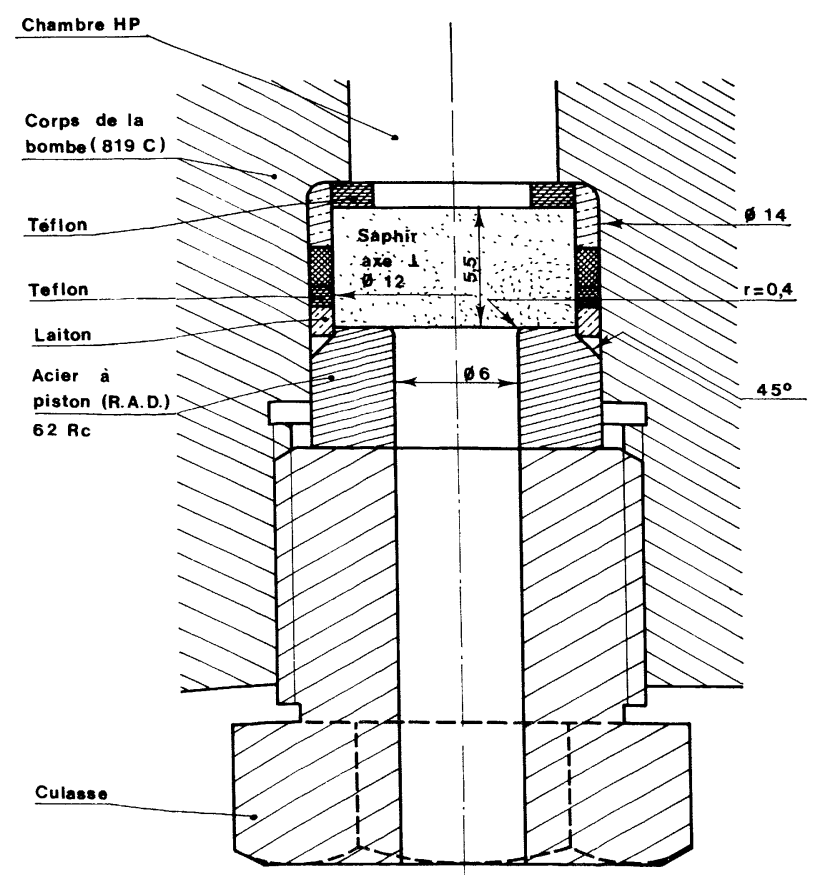

FIG. 2. - Dispositif avec joints à encastrement.

Pour ce qui est de la fenêtre, il faut noter deux points :

a) SES Dimensions. $-e=5,5 \mathrm{~mm}$ (épaisseur), $D=12 \mathrm{~mm}$ (diamètre total), $d=6 \mathrm{~mm}$ (diamètre utile de l'orifice).

Ceci donne des rapports $d / e=1,1$ et $d / D=0,50$.

b) L'ORientation de L'AXE. - Pour cet essai (Fig. 2), nous avons choisi d'orienter l'axe cristallin du saphir perpendiculairement à l'axe optique, contrairement à ce qui se fait souvent. Les saphirs utilisés sont des saphirs blancs synthétiques de qualité standard recuits après fabrication. L'élimination des contraintes par recuit est vérifiée entre polariseurs.

III. Résultats. - Le montage de la figure 1 nous a permis de travailler régulièrement à 14000 bars, et d'atteindre 16000 bars à titre d'essai. Après une dizaine de montées, aucune indentation n'est visible sur les fenêtres. Cet obturateur a été incorporé [2] dans un ensemble à haute pression d'hélium à $77^{\circ} \mathrm{K}$ qui a tenu 20 jours consécutifs à des pressions comprises entre 6 et 10 kilobars avec un taux de fuite en pression $\Delta P / P$ inférieur à $10^{-7}$ par minute. Ceci pour indiquer l'étanchéité réalisable avec le simple joint de Poulter, même sous pression d'hélium fluide.

Le deuxième type de montage (Fig. 2) a été essayé en tenue mécanique à 16 puis 17 kilobars de pression liquide (pentane). A 17 kilobars, le tube d'amenée à la bombe optique (capillaire d'acier inoxydable étiré à froid) a explosé. Après l'explosion, aucune marque n'était visible sur la fenêtre de saphir.

IV. Discussion des résultats. - 1) TENUE MÉCANIQUE DES FENÊTRES DE SAPHIR. - D'après les essais décrits il semble possible d'utiliser les fenêtres de saphir sous des contraintes beaucoup plus élevées qu'il n'est généralement admis, à notre connaissance. A titre d'exemple, Hughes et Robertson [3] utilisent des géométries $d / e=1$ et $d / D=0,4$ jusqu'à 6000 bars. Nous avons pu atteindre près du triple de cette pression pour des géométries plus " légères 》 encore $d / e=1,1$ et $d / D=0,50$. On peut noter à ce sujet qu'en tenant compte de l'indice de réfraction du saphir l'ouverture optique théorique totale est de plus de 100 degrés pour la fenêtre seule.

En second lieu, même à ces taux de contrainte n'apparaît pas encore la nécessité d'aligner l'axe optique du matériau avec l'axe géométrique de la fenêtre.

Enfin, le saphir semble supporter en milieu liquide (pentane) des vitesses de compression supérieures à 10 kilobars/minute, et la décompression explosive sans dommage apparent. Après l'explosion, nous avons cependant observé une déformation de 5 franges du siège et de 2 franges du saphir, ce qui est sans doute dû à la déformation du siège soumis, à 17000 bars à une contrainte normale de plus de 23000 bars.

2) JoIntS enCAStrés SUR La fenÊtre. - Les empilements de joints à bagues, chevauchant la fenêtre et l'obturateur optique ont été déjà utilisés [3] à notre connaissance jusqu'à 6000 bars. Ils donnent satisfaction sous une forme très légèrement différente jusqu'à 17000 bars au moins. L'explosion des fenêtres cristallines épaisses sous pression est engendrée dans de nombreux cas par des amorces sur la surface portante $[1,4]$. Sans discuter le mode de fonctionnement des joints à encastrement, on peut noter qu'aux pressions atteintes, le dernier joint de laiton est largement déformé sur le chanfrein. Par suite, la fenêtre est soumise à une surpression radiale (cerclage) par effet de coin. Il n'est pas impossible que ceci contribue à sa tenue.

IV. Conclusion. - Ce type de montage permet de réaliser des ensembles optiques beaucoup plus com- 
pacts que ceux proposés jusqu'ici (voir par exemple Réf. $[3,5,6])$. Le diamètre de l'ensemble optique n'est en effet, supérieur à celui de la fenêtre que de deux fois l'épaisseur des bagues, à la différence des obturateurs de Bridgman. La longueur axiale est, d'autre part, à peu près l'épaisseur de la fenêtre seule, épaisseur qui, nous le montrons, peut être inférieure au diamètre utile jusqu'à 17000 bars au moins.

\section{Bibliographie}

[1] Paul (W.), de Meis (W. M.), Besson (J. M.), Rev. Sci. Inst., 1968, 39, 928.

[2] Fournier (J.), Mémoire du C. N. A. M., Paris, juin 1969.

[3] Hughes (D. S.), Robertson (W. W.), J. Opt. Soc. Am., 1956, 46, 557.
[4] Warschauer (D. M.), Paul (W.), Rev. Sc. Inst., 1958, 29, 675 .

Besson (J. M.), Thèse, Paris, 1967.

[5] Fitchen (D. B.), Rev. Sci. Inst., 1963, 34, 673.

[6] Langer (D.), Warschauer (D. M.), Rev. Sci. Inst. 1961, 32, 32. 